Int. J. Biol. Chem. Sci. 11(1): 499-514, February 2017

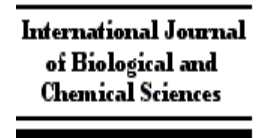

ISSN 1997-342X (Online), ISSN 1991-8631 (Print)

Original Paper http://ajol.info/index.php/ijbcs http://indexmedicus.afro.who.int

\title{
Caractérisation physico-chimique et géotechnique de deux sites argileux du Bénin en vue de leur valorisation dans l'éco-construction
}

\author{
Armel B. LAIBI ${ }^{1,2,4^{*}}$, Moussa GOMINA ${ }^{2}$, Brahima SORGHO ${ }^{3}$, \\ Etienne $\mathrm{SAGBO}^{4}$, Philippe BLANCHART ${ }^{5}$, Mohamed BOUTOUIL ${ }^{6}$ et \\ Dominique K. C. SOHOUNHLOULE ${ }^{1}$
}
${ }^{1}$ Laboratoire d'Etude et de Recherche en Chimie Appliquée, Ecole Polytechnique d'Abomey-Calavi, Université d'Abomey-Calavi (LERCA/EPAC/UAC), 01 BP 2009 Cotonou, Benin.
${ }^{2}$ Equipe Structure et Comportement Thermomécanique des Matériaux (ESTM) du Crismat, UMR 6508, Ensicaen, 6 boulevard du Maréchal Juin, 14050 Caen Cedex 4, France.
${ }^{3}$ Equipe de Physico-Chimie et de Technologie des Matériaux, Département de Chimie, Université de Ouagadougou, 03 BP 7021 Ouagadougou 03, Burkina Faso.
${ }^{4}$ Laboratoire de Chimie Inorganique et de l'Environnement (LACIE), Département de Chimie, Faculté des Sciences et Techniques Université d'Abomey-Calavi, 01 BP 4521 Cotonou, Bénin.
${ }^{5}$ SPCTS, UMR 7315 ENSCI 12 rue Atlantis, 87068 Limoges Cedex, France.
${ }^{6}$ Ecole d'Ingénierie et Travaux de la Construction de Caen, 14610, Epron, France.
*Auteur correspondant ; E-mail : armel.laibi@ensicaen.fr; Tel : (+33) 754577887.

\section{RESUME}

Dans le but de contribuer à la valorisation des matériaux argileux du Bénin dans l'éco-construction, dans ce travail, il a été question de procéder à la caractérisation géotechnique, physico-chimique, minéralogique et thermique des sols prélevés dans la région de Parakou (Baka) et de Ketou (Etigbo). Pour cela divers méthodes et moyens d'identification ont été utilisés, notamment les analyses géotechniques, les analyses minéralogiques, les analyses thermiques et l'analyse chimique. Il ressort de la caractérisation géotechnique que le sol de Baka est un limono-sableux avec un indice de plasticité IP $(\%)=14$ et le sol de Etigbo, un argilolimoneux avec un indice de plasticité IP $(\%)=38$. Ces résultats traduisent que les deux échantillons sont respectivement des sols de type A2 et A3. L'étude minéralogique couplée à l'étude chimique ont permis l'identification et la quantification des minéraux dont 78,18\% de kaolinite ; 13,20\% d'hématite; 7,06\% de quartz et $1,58 \%$ d'anatase pour le sol d'Etigbo et 33,46\% de Kaolinite ; $14,90 \%$ d'illite ; $2,11 \%$ de microcline ; $48,78 \%$ de quartz et $0,86 \%$ d'anatase pour le sol de Baka. Le comportement thermique entre $25^{\circ} \mathrm{C}$ et $1100{ }^{\circ} \mathrm{C}$ est principalement marqué par le rôle individuel des minéraux, de l'eau hygroscopique et de la matière organique présents dans les échantillons. Cette caractérisation indique que le sol de Baka convient pour la formulation des blocs de terre compressé (BTC) et le sol de Etigbo, pour la confection des céramiques réfractaires.

(C) 2017 International Formulae Group. All rights reserved.

Mots clés : Matériaux argileux, Bénin, caractérisation, limono-sableux, argilo-limoneux, blocs de terre compressé. 


\title{
Physicochemical and geotechnical characterization of two clayed sites of Benin in order for their volarization in eco-building
}

\begin{abstract}
In order to contribute to the development of Benin's clay materials in eco-building, we carried out geotechnical, physical, chemical, mineralogical and thermal characterization of soils taken from Parakou (Baka) and Ketou (Etigbo) areas in this work. Various methods and means of identification have been used, including geotechnical analyzes, mineralogical analyzes, Thermal analyzes and chemical analysis. According to the geotechnical characterization of soils, Baka soil is a sandy-silt with plasticity index PI (\%) $=14$ and Etigbo soil is a silty-clay with a plasticity index PI $(\%)=38$. These results reflect that both samples are soils type A2 and A3 respectively. The mineralogical study coupled with the chemical study enabled the identification and the quantification of minerals: $78.18 \%$ of kaolinite, $13.20 \%$ of hematite, $7.06 \%$ quartz and $1.58 \%$ of anatase for Etigbo soil and $33.46 \%$ of kaolinite, $14.90 \%$ of illite, $2.11 \%$ of microcline, $48.78 \%$ of quartz and $0.86 \%$ anatase for Baka soil. The thermal behavior between $25{ }^{\circ} \mathrm{C}$ and $1100{ }^{\circ} \mathrm{C}$ is mainly influenced by the individual role of minerals, hygroscopic water and organic material present in the samples. This characterization indicates that Baka soil is suitable for the formulation of compressed earth block (CEB) and Etigbo soil is appropriate for the manufacture of refractory ceramics.
\end{abstract}

(C) 2017 International Formulae Group. All rights reserved.

Keywords: Clay materials, Benin, characterization, sandy-silt, silty-clay, compressed earth blocks.

\section{INTRODUCTION}

Au Bénin, comme dans d'autres pays de la sous-région Ouest-africaine, la construction de maisons individuelles d'habitation obéit rarement aux réglementations techniques et administratives relatives à la qualité des matériaux à utiliser pour les fondations, les dimensions ou, la qualité des matériaux constitutifs des murs porteurs, etc (Laibi, 2008). Cela est illustré par les dégâts que causent chaque année les eaux de pluie aux habitations sur toute l'étendue du territoire et surtout en zones rurales (Laibi, 2008).

En zone périurbaine les matériaux traditionnels s'avèrent souvent inadaptés pour les constructions au format moderne, du fait de leurs performances limitées. Cela conduit à l'utilisation majoritaire de matériaux cimentaires qui présentent des inconvénients importants : coût énergétique pour la production du ciment, pollution induite, inconfort thermique des maisons en parpaings dans les zones sub-sahariennes, etc. Dans ce contexte, l'utilisation de blocs de terre comprimée (BTC) est une solution crédible et intéressante en cours d'expérimentation dans certains pays tels le Burkina Faso (Millogo, 2008; Sorgho, 2013), le Mali (Diawara, 2009; Keita, 2014), la Côte d'Ivoire (Emeruwa et al., 2008). L'utilisation de ce type de matériau s'insère convenablement dans une démarche visant à une haute qualité environnementale, puisque le procédé fait appel à un matériau abondant localement et ne nécessitant que peu d'énergie pour sa transformation (Ben Ayed, 2009). Néanmoins la terre comprimée ne peut pas être employée en construction que si sa cohésion est suffisante (Sorgho, 2013). Cette propriété essentielle étant principalement due à la présence d'argile qui joue le rôle de liant naturel. En conséquence l'utilisation des argiles dans les applications industrielles pour les BTC est tributaire de la connaissance détaillée de leurs propriétés physicochimiques et technologiques. En particulier, la plasticité des argiles, leur comportement lors du séchage et la résistance mécanique des 
produits après séchage ont aussi une grande importance (Camille, 2010).

Dans un souci de valorisation des matériaux utiles du Bénin, le présent travail est consacré à une caractérisation géotechnique, physico-chimique et thermique des sols prélevés dans la région de Parakou (Baka) et de Ketou (Etigbo) en vue de leur valorisation dans l'éco-construction (formulation des BTC).

\section{MATERIEL ET METHODES Matériel}

Les matières premières argileuses qui font l'objet de l'étude ont été prélevées dans les localités de Etikbo (commune de Ketou) (Figure 1) et de Baka (commune de Parakou) (Figure 2) au Bénin. Eigbo est une localité de la Commune de Kétou, située à l'extrémité Nord du département du Plateau (latitudes $7^{\circ}$ $10^{\prime}$ et $7^{\circ} 41^{\prime} 17^{\prime \prime}$ Nord et les longitudes $2^{\circ} 24^{\prime}$ $24^{\prime \prime}$ et $2^{\circ} 47^{\prime} 40^{\prime \prime}$ Est). Baka appartient à la Commune de Parakou (latitudes $9^{\circ} 00^{\circ}$ et $10^{\circ}$ $00^{\prime}$ Nord et les longitudes $2^{\circ} 20^{\prime}$ et $3^{\circ} 40^{\prime}$ Est). Les deux matières premières ont été référencées ETI pour Etigbo et BAK pour Baka.

Sur le plan géologique, la région de Kétou se situe sur la bordure Nord-Est du Bassin sédimentaire côtier, où affleurent des formations d'âge Maestrichtien (Crétacé supérieure), recouvertes localement de cuirasses latéritiques. La région de Parakou, quant à elle, repose sur un ancien socle antécambrien arasé, constitué de granites et de gneiss plus ou moins métamorphosés avec une tectonique peu évidente.

\section{Méthodes expérimentales}

Avant les analyses, les échantillons ont été concassés dans un mortier en agathe et tamisés à $2 \mathrm{~mm}$.

La détermination de la teneur en matières organiques a été effectuée par attaque à l'eau oxygénée (Staljanssens et al., 1975). La teneur en matières organiques $\left(\mathrm{C}_{\mathrm{MOC}}\right)$ contenues dans le sol est égale au rapport de la différence entre la masse initiale de l'échantillon $m_{0}$ et la masse de l'échantillon après la réaction $\mathrm{m}_{1}$ à la masse initiale d'essai $\mathrm{m}_{\mathrm{o}}$ (100 grammes).

$\mathrm{C}_{\text {MOC }}(\%)=100\left(\mathrm{~m}_{\mathrm{o}}-\mathrm{m}_{1}\right) / \mathrm{m}_{\mathrm{o}}$

La masse $m_{1}$ est obtenue après les opérations suivantes :

- pesé $100 \mathrm{~g}$ d'échantillon et le mettre dans un bécher de $500 \mathrm{ml}$;

- ajouté $200 \mathrm{~cm}^{3}$ d'eau distillée et $20 \mathrm{~cm}^{3}$ d'eau oxygénée, 10 volumes ;

- laissé reposer le mélange pendant 2 heures ;

- séché à l'étuve à $105^{\circ} \mathrm{C}$;

- pesé le mélange.

L'analyse granulométrique par tamisage humide et par sédimentométrie a été faite respectivement selon les normes NF P18560 (1978) et NF P94-057 (1992).

La limite de liquidité $\left(\mathrm{W}_{\mathrm{L}}\right)$ a été déterminée selon la méthode du disque de Casagrande et la limite de plasticité $\left(\mathrm{W}_{\mathrm{P}}\right)$ selon la méthode $\mathrm{du}$ rouleau. Ces limites d'Atterberg ont été déterminées en suivant la norme NF P94-051 (1993).

La détermination de la surface spécifique par la méthode du bleu de méthylène, qui est représentative de la teneur en argiles de l'échantillon, a été déterminée selon la norme NF P 94-068 (1993).

L'essai d'équivalent de sable qui rend compte de la quantité et de la qualité des phases granulaires les plus fines contenues dans une fraction, a été fait selon la norme NF EN 933-8 (1999).

La teneur en eau optimale $\left(\mathrm{W}_{\text {opt }}\right)$ et la densité sèche maximale $\left(\delta_{\text {opt }}\right)$ ont été déterminées par l'essai Proctor normal selon la norme NF P94-093 (1999). La teneur en eau optimale est un indice qui permet de caractériser le comportement d'une terre en présence de l'eau. Elle représente la quantité d'eau nécessaire pour lubrifier les particules de terre et leur permettre de se déplacer à l'intérieur de la masse afin d'occuper le moins de place possible.

Les compositions chimiques des échantillons ont été déterminées par spectrométrie à torche à plasma d'émission optique (ICP-OES (JY 38 Plus)). La composition minéralogique a été obtenue à 
partir de la diffraction des rayons $\mathrm{X}$ sur les poudres d'échantillons brutes, traitées par saturation à l'éthylène-glycol et chauffées à $490{ }^{\circ} \mathrm{C}$ pendant 4 heures. Le diffractomètre utilisé est un appareil de marque Bruker D 5000 avec un monochromateur arrière en graphite fonctionnant avec la radiation $\mathrm{CuK} \alpha$. Les diffractogrammes ont été traités par le logiciel X'Pert High Score Plus. A partir des résultats de l'analyse chimique et ceux de la diffraction des rayons $\mathrm{X}$, la composition semiquantitative des phases minérales de l'échantillon a été évaluée en utilisant la relation NJOPWOUO et al. (1979). Cette relation est la suivante :
$\mathrm{T}(\mathrm{a})=\Sigma \mathrm{Mi} \times \mathrm{Pi}(\mathrm{a})$, dans laquelle :

- $\mathrm{T}(\mathrm{a})=$ teneur $($ oxyde $\%$ ) en l'élément chimique $« \mathrm{a} »$;

- $\mathrm{Mi}=$ teneur $(\%)$ en minéral $« \mathrm{i} »$ dans la matière étudiée et contenant l'élément « $\mathrm{i} »$;

- Pi (a) = proportion de l'élément « a » dans le minéral « $\mathrm{i} »$.

Le comportement thermique des échantillons (Analyse Thermique Différentielle et Thermogravimétrique ATD/TG) ont été enregistrés à l'aide d'un appareil SETARAM réglé à une vitesse de montée de $10^{\circ} \mathrm{C} / \mathrm{min}$ avec un palier à $1100^{\circ} \mathrm{C}$ dans une atmosphère d'air.

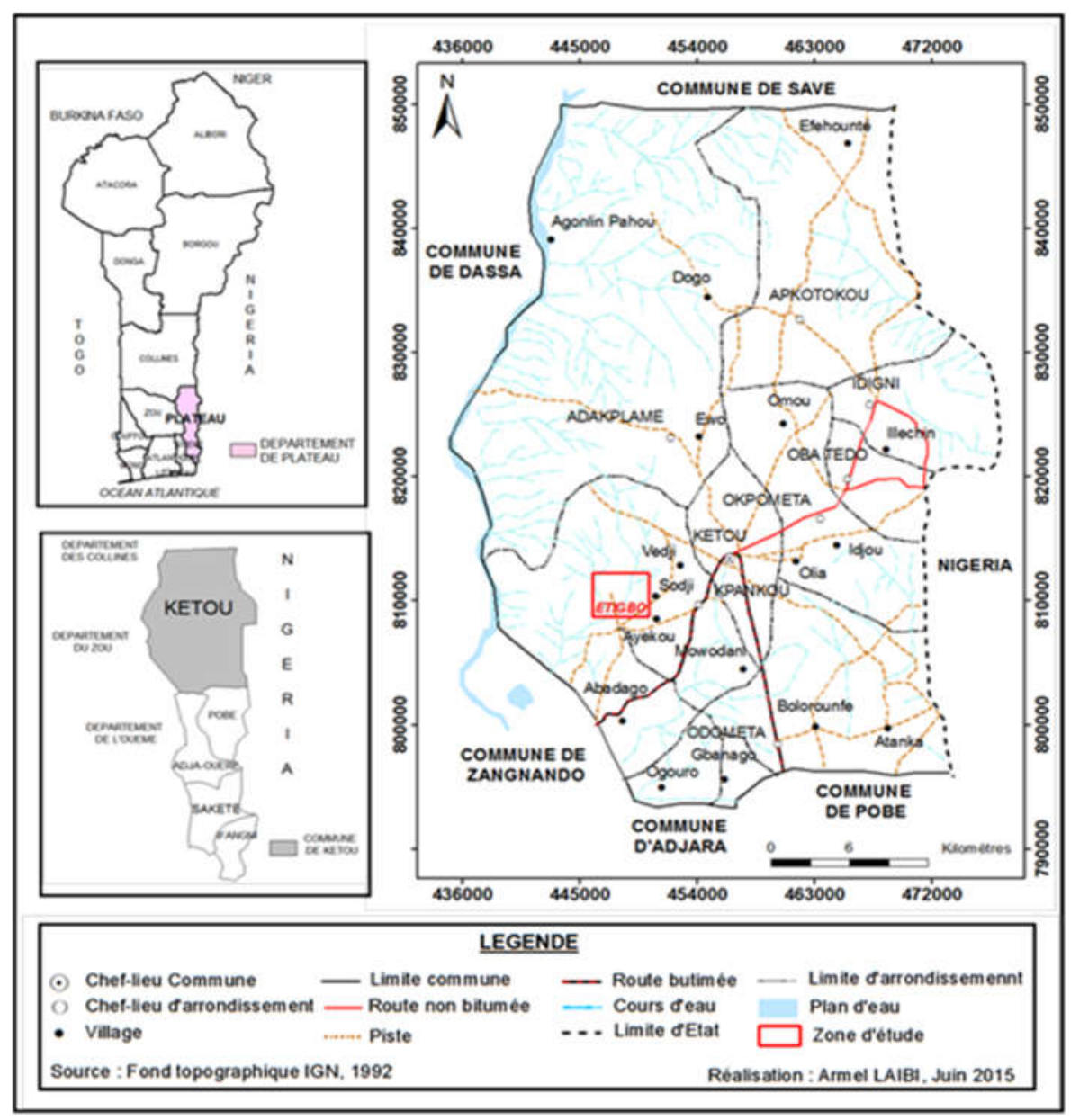

Figure 1 : Localisation du site de prélèvement de l'échantillon ETI. 


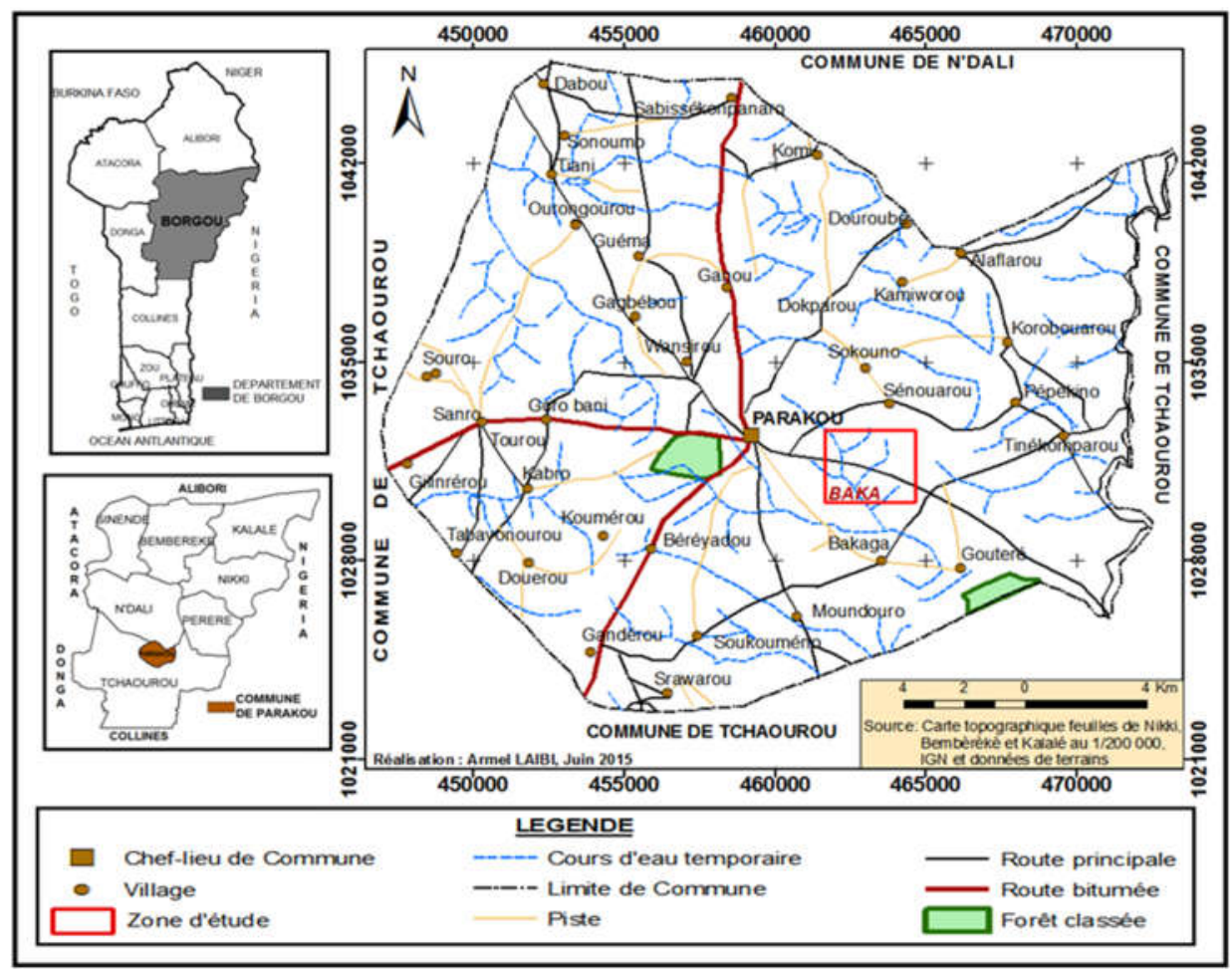

Figure 2: Localisation du site de prélèvement de l'échantillon BAK.

\section{RESULTATS}

\section{Caractérisations géotechniques}

Les teneurs en matières organiques des échantillons des deux sites étudiés sont présentées dans le Tableau 1. BAK a une teneur en matière organique inférieure à $2 \%$. C'est donc un sol très pauvre en matière organique qui peut être qualifié géotechniquement de sol inorganique (Ceratec, 1987; Guerraou et al., 2008). La teneur en matière organique de ETI est légèrement supérieure au seuil de $2 \%$ et est acceptable géotechniquement. Ces faibles teneurs indiquent que la matière organique aura un rôle négligeable dans les procédés de fabrication (stabilisation ou cuisson) des briques à partir des échantillons.

Le Tableau 2 présente la composition granulométrique de BAK, de ETI en comparaison des valeurs de matériaux de référence bien adaptés à la stabilisation par compaction. De l'analyse du Tableau 2, il ressort que BAK est un échantillon limonosableux et ETI, un échantillon argilolimoneux. En établissant une comparaison entre les échantillons et les sols de référence, BAK pourrait être éligible pour la confection des briques crues.

Les limites d'Atterberg aussi ont été déterminées et comparées à celle de matériaux utilisés pour réaliser les sols compactés. Le Tableau 3 présente une récapitulation des spécifications concernant les limites d'Atterberg.

Le Tableau 4 présente les résultats de l'essai au bleu de méthylène (VBS) et de l'équivalent de sable (ES).

Connaissant la nature des échantillons, la densité en eau optimale a été évaluée grâce à l'essai Proctor. ETI étant riche en fraction argileuse, il s'est révélé inapproprié à la réalisation de l'essai. La Figure 3 présente la courbe Proctor de BAK. 
Analyses

minéralogique

Analyse chimique

Le Tableau 5 présente les résultats de l'analyse chimique élémentaire de BAK et de ETI. De l'analyse de ces données, il ressort que la silice et l'alumine sont les oxydes majeurs dans les deux échantillons, traduisant qu'ils sont des aluminosilicates.

\section{Analyses minéralogiques}

Les Figures 4 et 5 présentent respectivement les diffractogrammes des échantillons de BAK et de ETI. Le dépouillement du diffractogramme de BAK (Figure 4) révèle qu'il est composé de kaolinite $\left(2 \mathrm{SiO}_{2}, \mathrm{Al}_{2} \mathrm{O}_{3}, 2 \mathrm{H}_{2} \mathrm{O}\right)$, d'illite $\left(\mathrm{K}_{2} \mathrm{O}, 2 \mathrm{H}_{2} \mathrm{O}, 2 \mathrm{Al}_{2}\left(\mathrm{Si}_{3} \mathrm{Al}\right) \mathrm{O}_{10}(\mathrm{OH})\right.$, auxquels sont associés du quartz $\left(\mathrm{SiO}_{2}\right)$, de l'anatase $\left(\mathrm{TiO}_{2}\right)$ et de la microcline $\left(\mathrm{KAlSi}_{3} \mathrm{O}_{8}\right)$. ETI (Figure 5) est essentiellement constitué de kaolinite $\left(2 \mathrm{SiO}_{2}, \mathrm{Al}_{2} \mathrm{O}_{3}, 2 \mathrm{H}_{2} \mathrm{O}\right)$, d'hématite $\left(\mathrm{Fe}_{2} \mathrm{O}_{3}\right)$, d'anatase $\left(\mathrm{TiO}_{2}\right)$ et de quartz $\left(\mathrm{SiO}_{2}\right)$. La première réflexion de l'illite (001) à $10 \AA$ n'est pas affectée ni par le traitement à l'éthylène-glycol, ni par le traitement thermique (Figures 6 et 7) tandis que les raies de la kaolinite ont disparu avec le chauffage à $490{ }^{\circ} \mathrm{C}$.

\section{Analyses thermiques différentielles et thermogravimétriques (ATD/TG)}

Les Figures 8 et 9 présentent respectivement les courbes des analyses thermiques différentielles et thermogravimétriques (ATG/TG) de BAK et de ETI. On voit que ces thermogrammes présentent des similitudes, puisque 3 pics endothermiques ont été mis en évidence sur les thermogrammes des deux échantillons et 3 pics exothermiques sur celui de BAK et 2 pics sur celui de ETI.

\section{DISCUSSION}

Le Tableau 3 montre que BAK a une plasticité moyenne (IP $=14 \%)$ et est donc apte pour la confection des blocs de terre comprimée (BTC). Selon le Guide de
Terrassement Routière (GTR), le traitement à la chaux est nécessaire avec des sols de type A3 et A4 c'est-à-dire des sols ayant une limite de liquidité importante et un indice de plasticité supérieur à 20 (Guerraoui et al., 2008). BAK ne peut donc pas être stabilisé avec de la chaux et cela est en accord avec les résultats du Tableau 2. En ce qui concerne ETI, son indice de plasticité indique qu'il peut être stabilisé avec la chaux. Néanmoins, les résultats de l'analyse granulométrique (Tableau 2), sont contradictoires puisqu'ils indiquent qu'il ne peut être utilisé pour la fabrication de BTC amendés ou non. D'après les valeurs de VBS, BAK est un sol limoneuxsableux et ETI, un sol argilo-limoneux comme déjà mis en évidence par l'analyse granulométrique. Par combinaisons des résultats des limites d'Atterberg et ceux de VBS, et selon la classification de GTR, BAK est un sol de type A2 et ETI, un sol de type A3. BAK est donc apte à être utilisé dans la confection des BTC contrairement à ETI qui peut être valorisé dans le domaine de la céramique.

Connaissant la nature des échantillons, la densité en eau optimale a été évaluée grâce à l'essai Proctor. ETI étant riche en fraction argileuse, il s'est révélé inapproprié à la réalisation de l'essai. La Figure 3 présente la courbe Proctor de BAK.

D'après la Figure 3, la masse volumique optimale $\left(\delta_{\text {opt }}\right)$ de BAK est de 1846 $\mathrm{kg} / \mathrm{m}^{3}$ et la teneur en eau optimale $\left(\mathrm{W}_{\text {opt }}\right)$ est de $12 \%$. Une argile pure a une courbe Proctor plus allongée et une $\mathrm{W}_{\text {opt }}$ plus élevée. La forme de la courbe Proctor de BAK est corrélée aux limites d'Atterberg (Tableau 3), avec une limite de liquidité de $28 \%$, une limite de plasticité de $14 \%$ et un indice de plasticité ( $\left.\mathrm{I}_{\mathrm{P}}\right)$ de $14 \%$. La densité $\delta_{\text {opt }}$ de ce sol étant compris entre 1760 et $2100 \mathrm{Kg} / \mathrm{m}^{3}$, il est donc qualifié de sol à malaxage satisfaisant.

De façon générale, le rapport silice/alumine est un indicateur de la teneur d'un échantillon en minéraux argileux et ce rapport est de 4,73 pour l'échantillon de 
BAK. Il peut être comparé à celui des minéraux argileux de type 2:1, qui du fait des nombreuses substitutions possibles, est compris entre 2 et 4 (Jouennec, 1990; Konank, 2006). Cette valeur élevée suggère la présence d'une quantité importante de silice libre dans BAK, et cela est corrélé aux résultats d'analyses granulométriques (Tableau 2). Le rapport Silice/Alumine est égal à 1,2 pour ETI, et est très proche de celui des kaolins purs $(1,1)$ (Yah et al., 1979). Le taux élevé d'alumine et la faible teneur en éléments alcalins $\left(\mathrm{K}_{2} \mathrm{O}\right.$ et $\left.\mathrm{Na}_{2} \mathrm{O}\right)$ indique la possibilité d'être utilisé comme matière première pour la confection de produits réfractaires (Ledoussalh, 1985; Sagbo et al., 2015). ETI se caractérise aussi par sa teneur particulièrement élevée en $\mathrm{Fe}_{2} \mathrm{O}_{3}(13,20 \%)$ qui induit une couleur rouge brique. La coloration intense est un atout pour cet échantillon car il peut être utilisé comme colorant naturel pour les blocs de terre stabilisée et les tuiles (Amoha, 2006).

ETI a une perte au feu $(13,91 \%)$ relativement élevée ce qui suggère aussi que sa teneur en phases argileuses est plus élevée que celle de BAK. BAK est un mélange kaolinite-illite avec une teneur élevée en quartz. On est donc en présence d'un mélange naturel semblable aux mélanges rencontrés en céramique silicatée avec lesquels du quartz est souvent ajouté aux minéraux argileux. Dans le cas des blocs de terres comprimées, l'illite et la kaolinite sont des minéraux importants dans leurs compositions puisqu'ils ne favorisent pas un phénomène de retrait/gonflement lors de séchage et de la réhumidification. La kaolinite est une espèce minérale réfractaire qui limite le fluage à haute température et est dominante dans ETI. Cette forte teneur en kaolinite est recherchée dans le domaine des céramiques réfractaires.

Le rapprochement des résultats de la diffraction des rayons $\mathrm{X}$ à ceux de l'analyse chimique élémentaire et en utilisant la relation de NJOPWOUO et al. (1979) a permis d'évaluer les quantités relatives des minéraux contenues dans les deux matières premières locales (Tableau 6). La composition minéralogique révèle que l'échantillon $\mathrm{BAK}$ a une forte teneur en Quartz $(49,4 \%)$ et en kaolinite $(33,1 \%)$. On en déduit que le quartz est la source de silice libre comme indiqué précédemment avec le calcul du rapport $\mathrm{SiO}_{2} / \mathrm{Al}_{2} \mathrm{O}_{3}$. L'échantillon ETI a une teneur élevée en kaolinite $(78,1 \%)$ comme le confirme les valeurs du rapport $\mathrm{SiO}_{2} / \mathrm{Al}_{2} \mathrm{O}_{3}$. Il a aussi une teneur importante en hématite $\mathrm{Fe}_{2} \mathrm{O}_{3}(13,2 \%)$ qui a un rôle de colorant. Les fortes teneurs en quartz ont un rôle de dégraissant de la kaolinite. Cela favorise l'utilisation de la matière première de $\mathrm{BAK}$ dans les formulations des éco-matériaux tels que les briques, les tuiles, etc. ETI a une composition favorable aux formulations des produits céramiques tels que les carreaux étant donné sa forte teneur en kaolinite.

Les valeurs de températures des pics ainsi que la nature des phénomènes associés sont reportés dans les Tableaux 7 et 8 . Dans le cas de BAK et aux températures inférieures à $200{ }^{\circ} \mathrm{C}$ l'analyse révèle que la combustion de la matière organique crée une perte de masse de $1,54 \%$. Cette valeur est relativement proche de celle déterminée $(1,33 \%)$ à partir de la norme XP P 94-047 (Tableau 1). Aux mêmes températures, le thermogramme de ETI indique une perte de masse de $12,02 \%$ (Tableau 8) attribuable à plusieurs phénomènes y compris la combustion de la matière organique.

L'absence du pic endothermique de la transformation allotropique du quartz sur le thermogramme de ETI (Figure 9) confirme la faible teneur du quartz décelée dans la minéralogie. Ces thermogrammes confirment que BAK contient de la kaolinite et du quartz et que ETI contient majoritairement de la kaolinite.

$$
\begin{aligned}
& \mathrm{Si}_{2} \mathrm{O}_{5} \mathrm{Al}_{2}(\mathrm{OH})_{4} \rightarrow 2 \mathrm{SiO}_{2}-\mathrm{Al}_{2} \mathrm{O}_{3}+2 \mathrm{H}_{2} \mathrm{O} \\
& \text { Kaolinite Métakaolinite } \\
& 2\left[2 \mathrm{SiO}_{2}-\mathrm{Al}_{2} \mathrm{O}_{3}\right] \rightarrow \mathrm{Si}_{3} \mathrm{Al}_{4} \mathrm{O}_{12}+\mathrm{SiO}_{2}
\end{aligned}
$$$$
\text { Métakaolinite Spinelle Silice amorphe }
$$ 
Tableau 1 : Les teneurs en matières organiques de BAK et de ETI.

\begin{tabular}{lllll}
\hline Echantillon & $\mathbf{m}_{\mathbf{0}}(\mathbf{g})$ & $\mathbf{m}_{\mathbf{1}}(\mathbf{g})$ & $\mathbf{m}_{\mathbf{2}}(\mathbf{g})$ & $\mathbf{C}_{\mathbf{M O C}}(\mathbf{\%})$ \\
\hline BAK & $248,93 \pm 10,05$ & $300,63 \pm 8,50$ & $299,93 \pm 8,61$ & $1,33 \pm 0,31$ \\
ETI & $220,92 \pm 9,98$ & $270,6 \pm 10,11$ & $269,45 \pm 10,12$ & $2,67 \pm 0,04$ \\
\hline
\end{tabular}

Tableau 2 : Compositions granulométriques de BAK, de ETI ainsi que de matériaux de référence pouvant atteindre une bonne stabilisation.

$\begin{array}{ccccc}\text { Echantillon } & \begin{array}{c}\text { Produits } \\ \text { finis }\end{array} & \text { Fractions granulométriques } & \begin{array}{c}\text { Stabilisants } \\ \text { (principaux) }\end{array} & \begin{array}{c}\text { Référence } \\ \text { bibliographique }\end{array}\end{array}$

Sols de référence

ETI $\checkmark \quad$ Argile : 15 à $25 \%$

$\checkmark$ Limon : 20 à $30 \%$

Briques $\quad \checkmark \quad$ Sable : 45 à $65 \%$

crues $\quad \checkmark$ Optimal : (Argile : 20\%

Limon : $22 \%$ Sable : $58 \%$ )

(Daot et al, 1991)

(4- $6 \%$ ou $8 \%$ )

Briques

crues $\quad \checkmark \quad$ Argile : $35 \%$

stabilisées $\quad \checkmark \quad$ sable fin : $25 \%$

$\checkmark$ sable grossier : $25 \%$

$\checkmark$ gravier fin $15 \%$

$30 \%$

Sable grossier : $30 \%$
Gravier fin $25 \%$

(Wetshondo, 2012)

Chaux

$(5-8 \%$ ou $12 \%)$

$\checkmark \quad$ Argile : $14,8 \%$

$\checkmark$ Limon : $23,5 \%$

$\checkmark$ Sable : $61,7 \%$

Argile : 43,5\%

Limon : 53,5\%

Sable : 3\% 
Tableau 3 : Limites d'Atterberg de BAK et de ETI.

\begin{tabular}{cccccc}
$\begin{array}{c}\text { Limites } \\
\text { d'Atterberg }\end{array}$ & BAK & ETI & $\begin{array}{c}\text { Zone limite } \\
\text { pour une BTC }\end{array}$ & $\begin{array}{c}\text { Zone } \\
\text { préférentielle } \\
\text { pour une BTC }\end{array}$ & $\begin{array}{c}\text { Référence } \\
\text { bibliographique }\end{array}$ \\
\hline $\mathrm{W}_{\mathrm{L}}(\%)$ & $\mathbf{2 8}$ & $\mathbf{6 8}$ & 25 à 50 & 30 à 35 & \\
$\mathrm{~W}_{\mathrm{P}}(\%)$ & $\mathbf{1 4}$ & $\mathbf{3 0}$ & 10 à 25 & 12 à 22 & $\begin{array}{c}\text { (Daot et al, } \\
1991)\end{array}$ \\
$\mathrm{I}_{\mathrm{P}}(\%)$ & $\mathbf{1 4}$ & $\mathbf{3 8}$ & 7 à 29 & 7 à 18 & \\
\hline
\end{tabular}

Tableau 4 : Equivalent de sable (ES) et Essai au bleu de méthylène (VBS) de BAK et de ETI.

\begin{tabular}{cccc}
\hline Echantillon & ES & VBS (g/100g) & Classification \\
\hline BAK & 13 & 1,1 & sol sablo-limoneux \\
ETI & 3 & 3,2 & $\begin{array}{c}\text { sol limoneux-argileux de plasticité } \\
\text { moyenne }\end{array}$ \\
\hline
\end{tabular}

Tableau 5 : Analyses chimiques (\% massique) de BAK et de ETI.

\begin{tabular}{|c|c|c|c|c|c|c|c|c|c|c|c|c|}
\hline Echantillon & $\begin{array}{c}\mathrm{SiO}_{2} \\
(\%)\end{array}$ & $\begin{array}{c}\mathrm{Al}_{2} \mathrm{O}_{3} \\
(\%)\end{array}$ & $\begin{array}{c}\mathrm{Fe}_{2} \mathrm{O}_{3} \\
(\%)\end{array}$ & $\begin{array}{c}\text { MnO } \\
(\%)\end{array}$ & $\begin{array}{c}\text { MgO } \\
(\%)\end{array}$ & $\begin{array}{c}\mathrm{CaO} \\
(\%)\end{array}$ & $\begin{array}{c}\mathrm{Na}_{2} \mathrm{O} \\
(\%)\end{array}$ & $\begin{array}{c}\mathrm{K}_{2} \mathrm{O} \\
(\%)\end{array}$ & $\begin{array}{l}\mathrm{TiO}_{2} \\
(\%)\end{array}$ & $\begin{array}{c}\mathrm{P}_{2} \mathrm{O}_{5} \\
(\%)\end{array}$ & $\begin{array}{l}\text { PF } \\
(\%)\end{array}$ & $\begin{array}{c}\text { Total } \\
(\%)\end{array}$ \\
\hline BAK & 72,01 & 15,20 & 3,01 & 0,01 & 0,30 & 0,31 & 0,23 & 1,58 & 0,77 & $<$ L.D. & 6,99 & 100,41 \\
\hline ETI & 39,37 & 30,90 & 13,20 & 0,02 & 0,10 & $<$ L.D. & 0,03 & 0,21 & 1,58 & 0,24 & 13,91 & 99,56 \\
\hline
\end{tabular}

Tableau 6 : Composition minéralogique de BAK et de ETI.

\begin{tabular}{ccccccc}
\hline Echantillons & $\begin{array}{c}\text { Kaolinite } \\
(\%)\end{array}$ & $\begin{array}{c}\text { Illite } \\
(\%)\end{array}$ & $\begin{array}{c}\text { Quartz } \\
(\%)\end{array}$ & $\begin{array}{c}\text { Microcline } \\
(\%)\end{array}$ & $\begin{array}{c}\text { Hématite } \\
(\%)\end{array}$ & $\begin{array}{c}\text { Anatase } \\
(\%)\end{array}$ \\
\hline BAK & $\mathbf{3 3 , 1}$ & $\mathbf{1 3 , 7}$ & $\mathbf{4 9 , 4}$ & $\mathbf{3 , 0}$ & - & $\mathbf{0 , 8}$ \\
ETI & $\mathbf{7 8 , 2}$ & - & $\mathbf{7 , 0}$ & - & 13,2 & 1,6 \\
\hline
\end{tabular}


Tableau 7 : Analyses thermiques de BAK.

\begin{tabular}{|c|c|c|c|c|}
\hline Nature du pic & $\begin{array}{c}\text { Température } \\
\left({ }^{\circ} \mathbf{C}\right)\end{array}$ & $\begin{array}{c}\text { Perte de } \\
\text { masse }(\%)\end{array}$ & Observations & $\begin{array}{c}\text { Référence } \\
\text { bibliographique }\end{array}$ \\
\hline Endothermique & 71 & 3,16 & Perte d'eau hygroscopique & \\
\hline Exothermique & 313 & 1,54 & $\begin{array}{c}\text { Combustion de la matière } \\
\text { organique }\end{array}$ & $\begin{array}{l}\text { (Millogo, 2008; } \\
\text { Rollet et al, 1972) }\end{array}$ \\
\hline \multirow[t]{2}{*}{ Endothermique } & 508 & & $\begin{array}{l}\text { Déshydroxylation de la } \\
\text { kaolinite (Equation I) }\end{array}$ & $\begin{array}{l}\text { [Sorgho, 2013; } \\
\text { Chenc et al, 2000) }\end{array}$ \\
\hline & 580 & & $\begin{array}{c}\text { Passage du quartz } \alpha \text { au } \\
\text { quartz } \beta\end{array}$ & (Sorgho, 2013) \\
\hline Exothermique & 952 & 7,47 & $\begin{array}{l}\text { Formation de mullite ou de } \\
\text { spinelle (Equation II) }\end{array}$ & $\begin{array}{l}\text { (Sorgho, 2013 ; } \\
\text { Soron, 2003) }\end{array}$ \\
\hline Phase vitreuse & 1171 & & $\begin{array}{l}\text { Formation d'une deuxième } \\
\text { génération de mullite }\end{array}$ & (Soron, 2003) \\
\hline
\end{tabular}

Tableau 8 : Analyses thermiques de ETI.

\begin{tabular}{|c|c|c|c|c|}
\hline Nature du pic & Température $\left({ }^{\circ} \mathbf{C}\right)$ & Perte de masse $(\%)$ & Observations & $\begin{array}{c}\text { Référence } \\
\text { bibliographique }\end{array}$ \\
\hline Endothermique & 56,22 & 1,26 & $\begin{array}{l}\text { Perte de l'eau } \\
\text { hygroscopique }\end{array}$ & \\
\hline Exothermique & 326,58 & & $\begin{array}{l}\text { Combustion de la } \\
\text { matière organique }\end{array}$ & $\begin{array}{l}\text { (Millogo, 2008; } \\
\text { Rollet et al, 1972) }\end{array}$ \\
\hline Endothermique & 513 & 12,02 & $\begin{array}{c}\text { Déshydroxylation de } \\
\text { la kaolinite et } \\
\text { formation de la } \\
\text { metakaolinite } \\
\text { (Equation I) }\end{array}$ & $\begin{array}{c}\text { [Sorgho, 2013; } \\
\text { Chenc et al, 2000) }\end{array}$ \\
\hline Exothermique & 985 & & $\begin{array}{l}\text { Formation de mullite } \\
\text { ou de spinelle } \\
\text { (Equation II) }\end{array}$ & (Sorgho, 2013) \\
\hline Phase vitreuse & 1212 & & $\begin{array}{c}\text { Formation d' } \\
\text { une deuxième } \\
\text { génération de mullite }\end{array}$ & $\begin{array}{l}\text { (Sorgho, } 2013 \text {; } \\
\text { Soron, 2003) }\end{array}$ \\
\hline
\end{tabular}


A. B. LAIBI et al. / Int. J. Biol. Chem. Sci. 11(1): 499-514, 2017

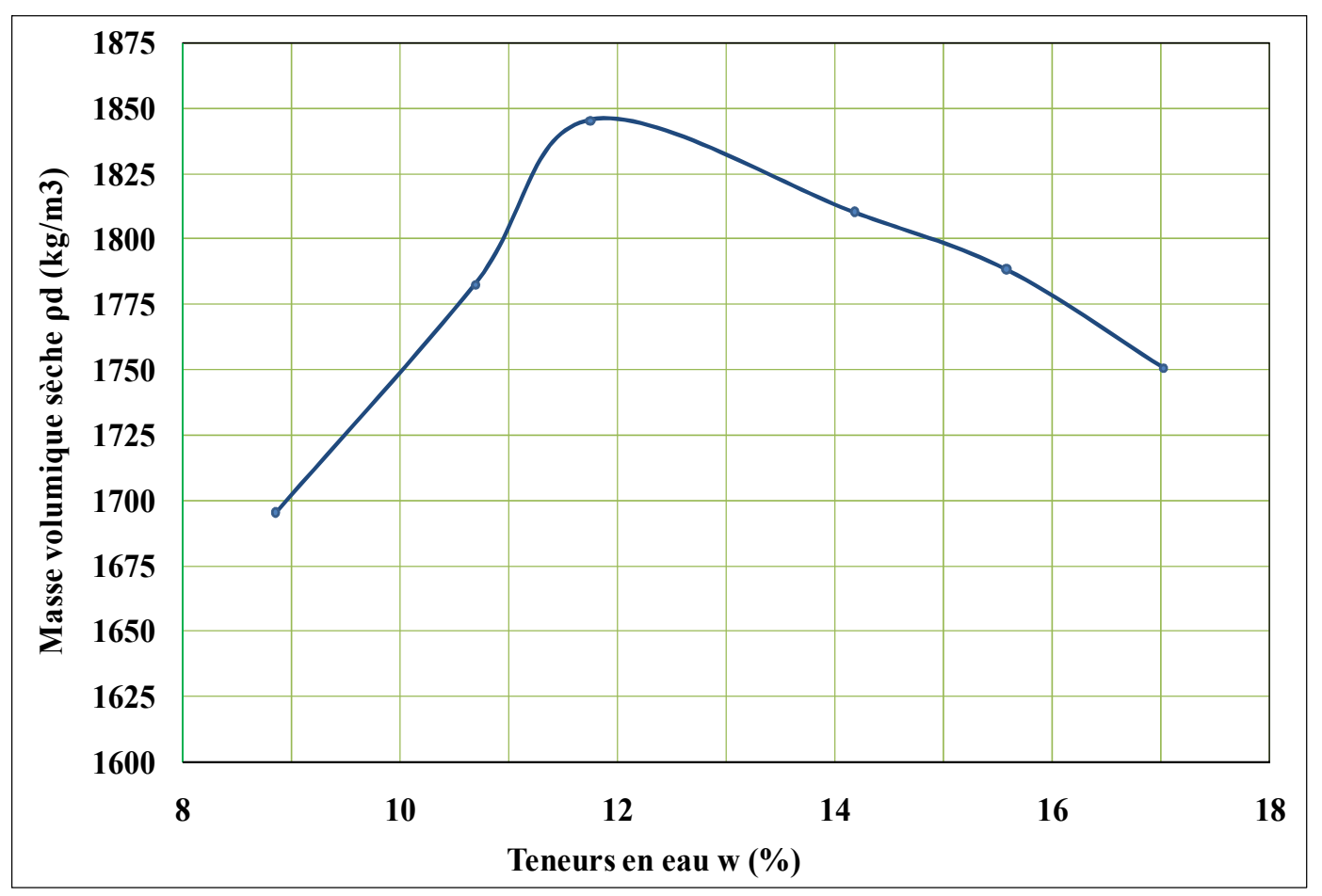

Figure 3 : Courbe Proctor de BAK.

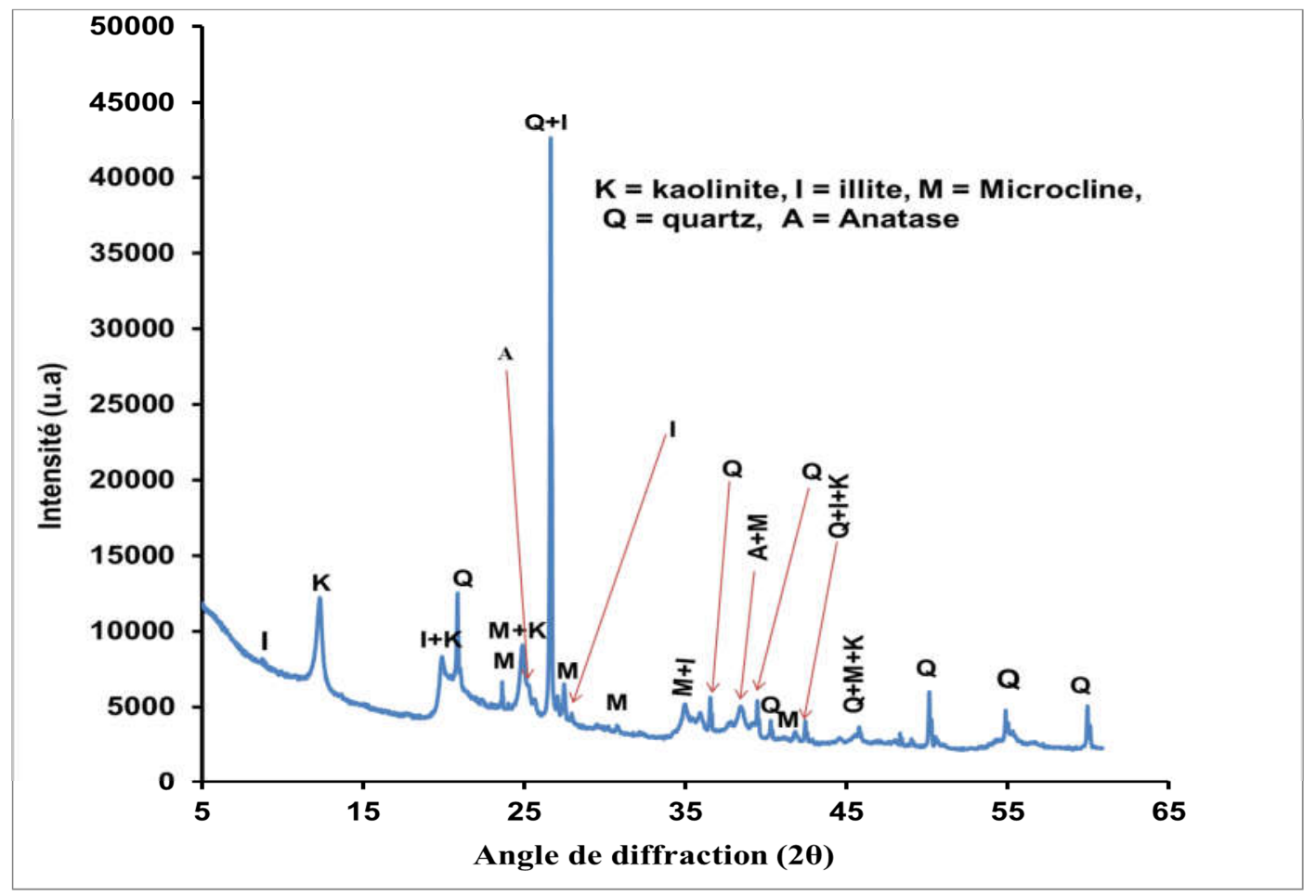

Figure 4 : Diffractogramme de BAK. 


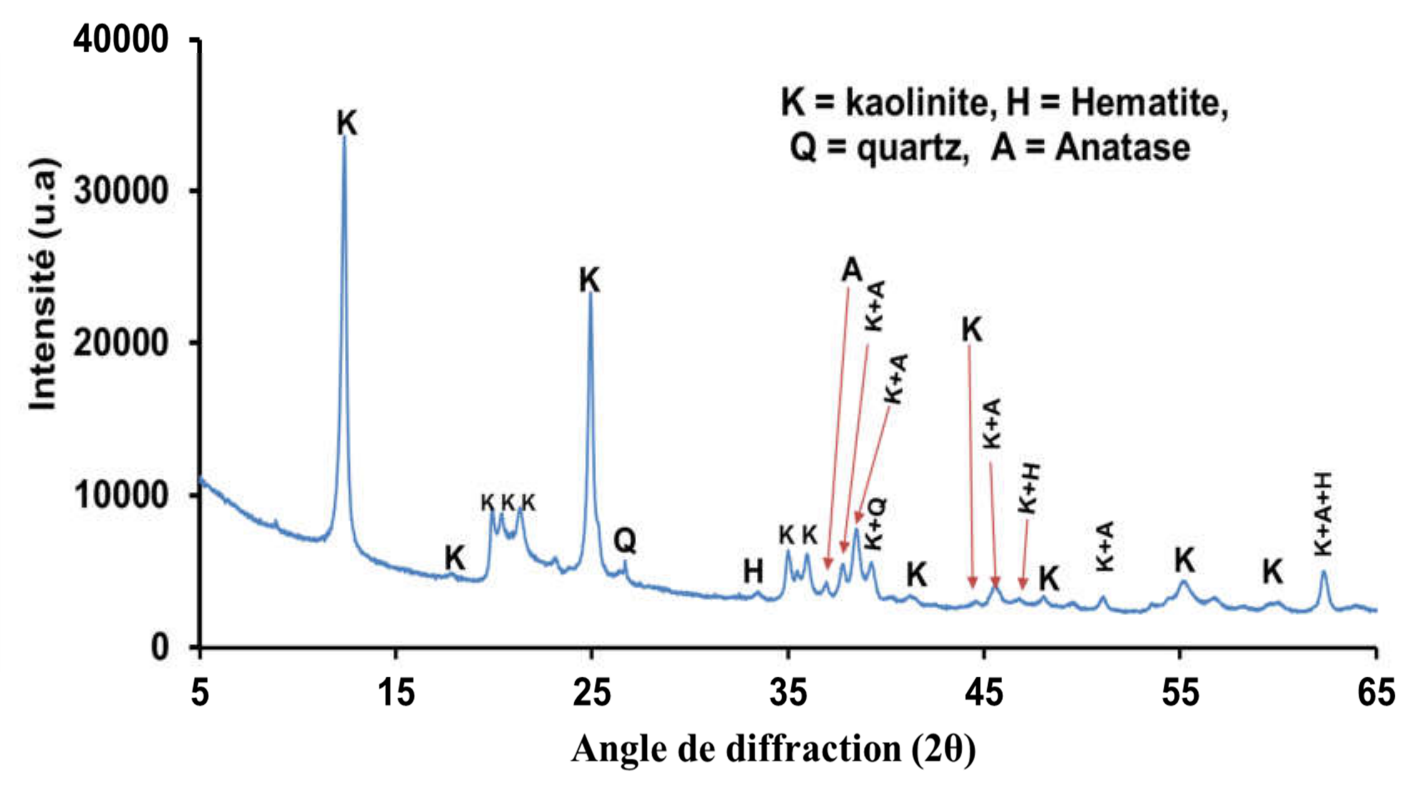

Figure 5 : Diffractogramme de ETI.

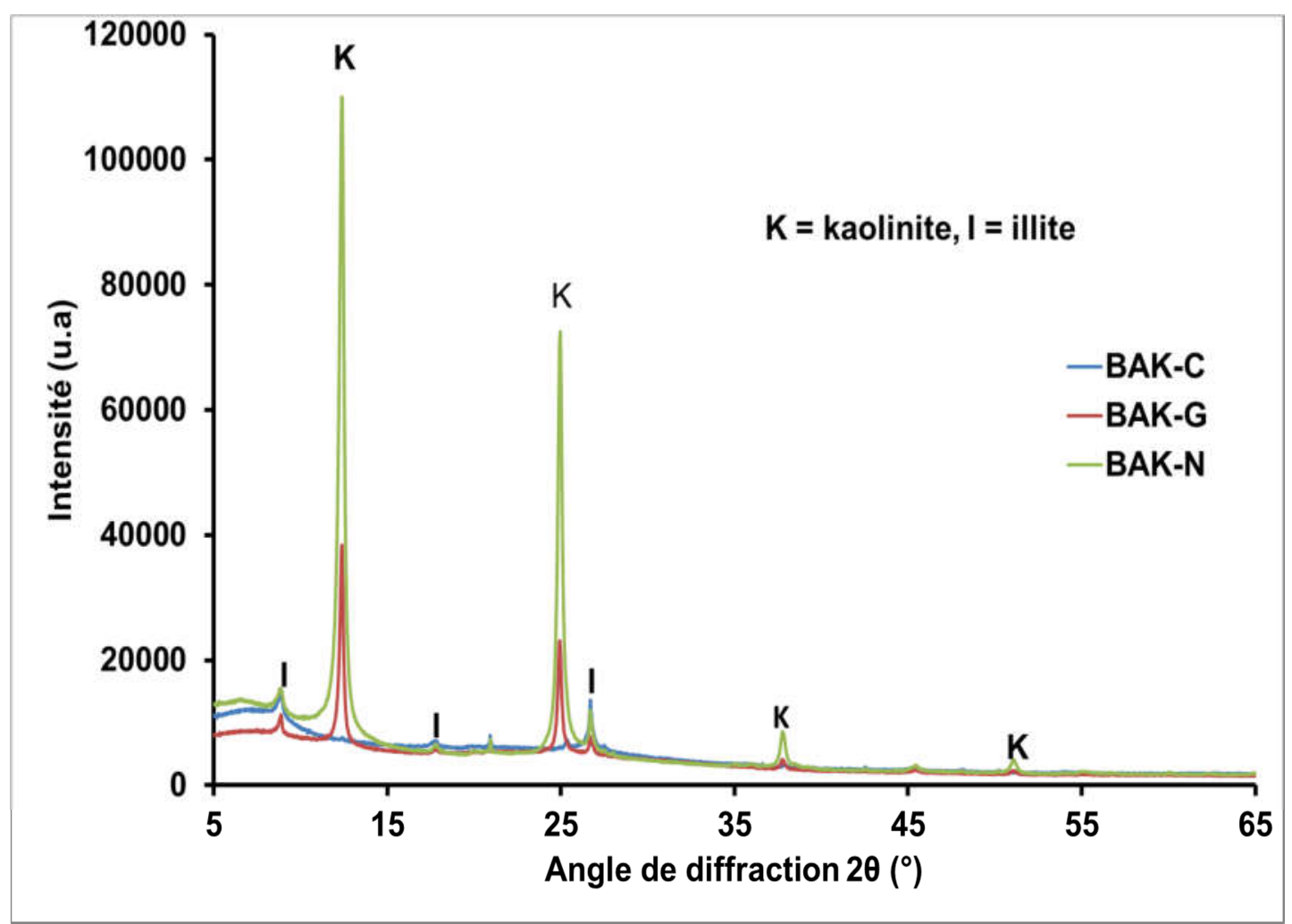

Figure 6 : Diffractogrammes de la fraction Normal $(\mathrm{N})$, traitée à éthylène-glycol $(\mathrm{EG})$, chauffée à $490{ }^{\circ} \mathrm{C}(\mathrm{C})$ de BAK. 


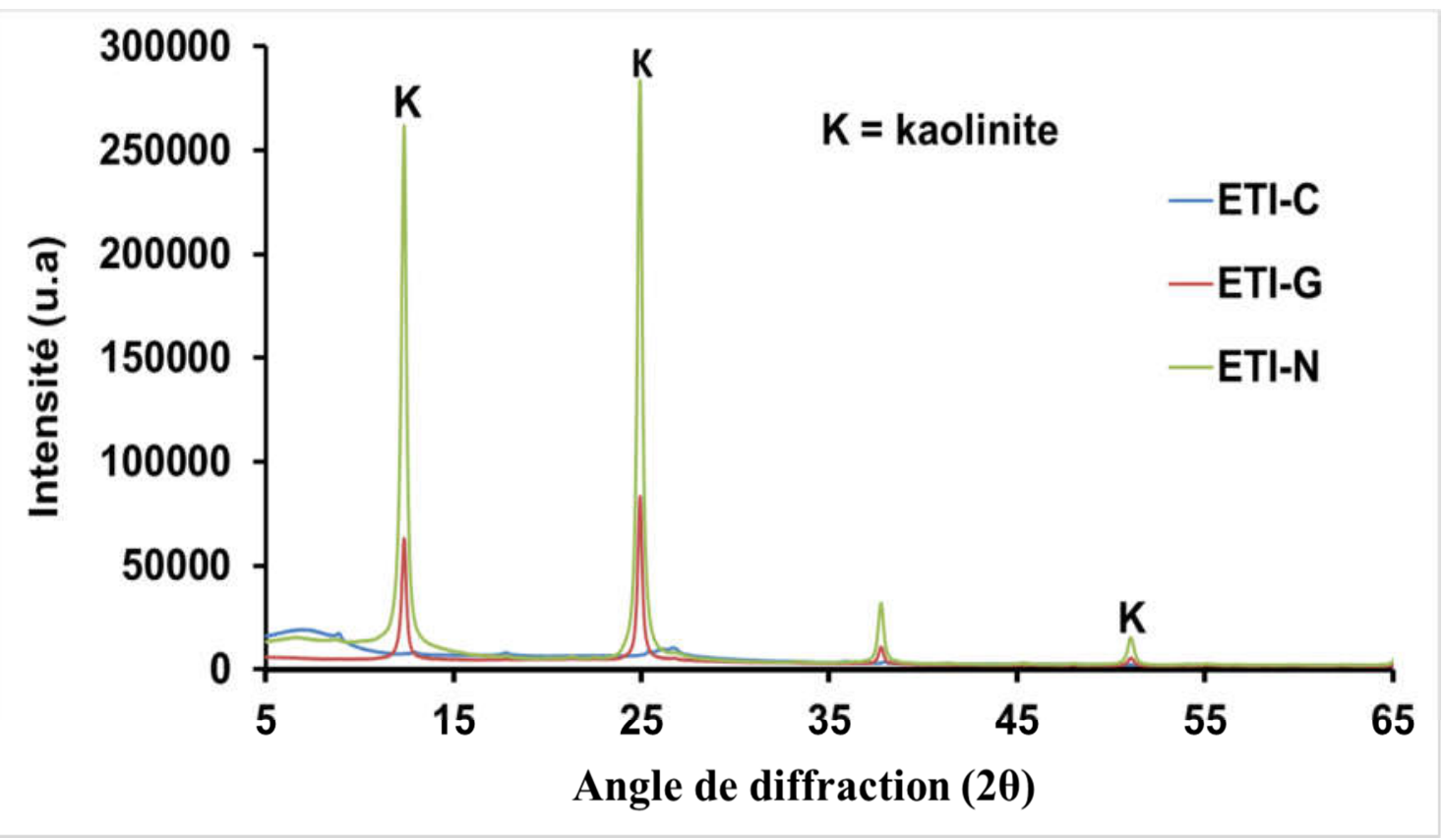

Figure 7 : Diffractogrammes de la fraction Normale $(\mathrm{N})$ de ETI, traitée à l'éthylène-glycol (EG) et chauffée à $490{ }^{\circ} \mathrm{C}(\mathrm{C})$.

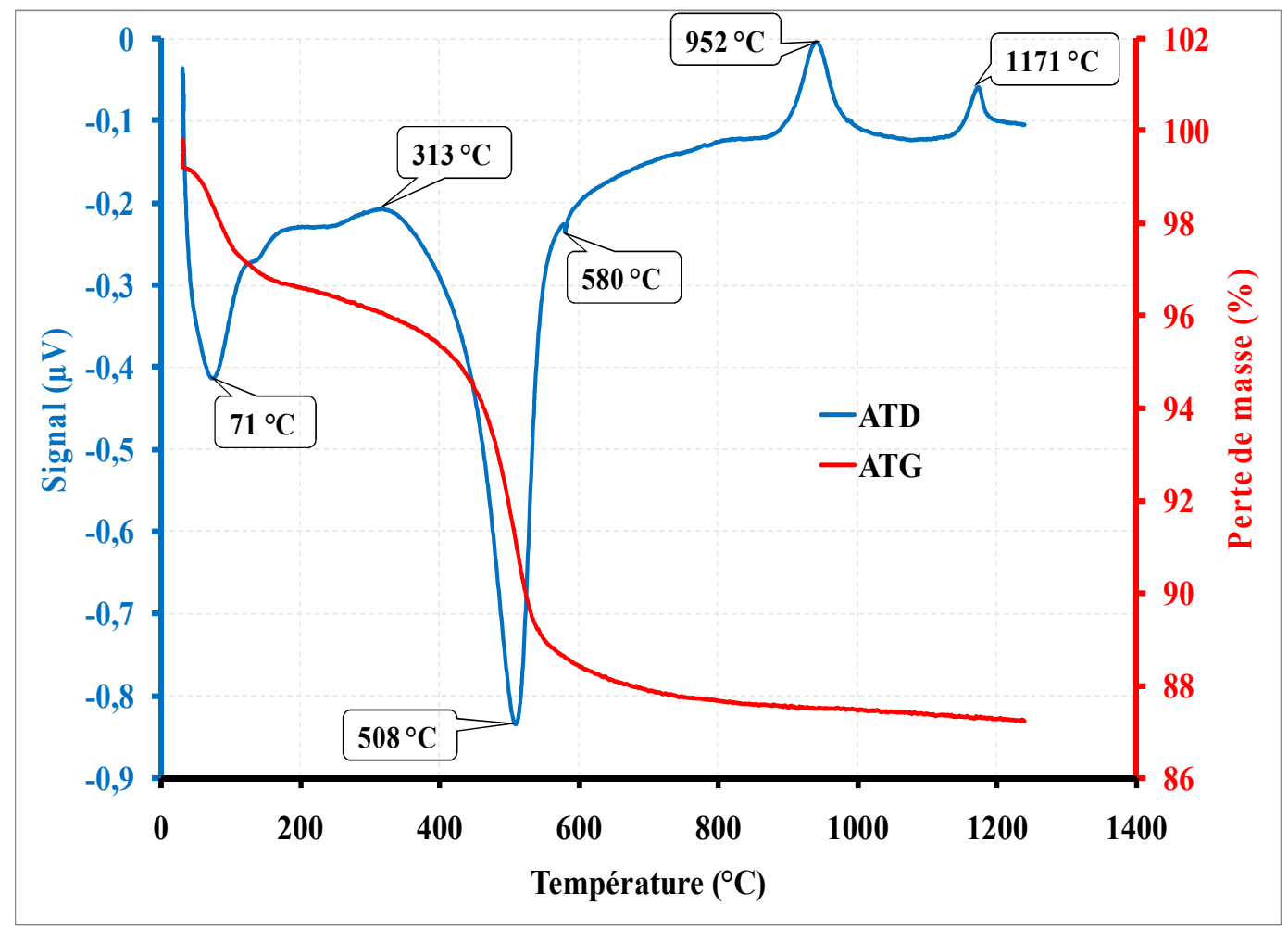

Figure 8 : Thermogrammes ATD/TG de BAK. 


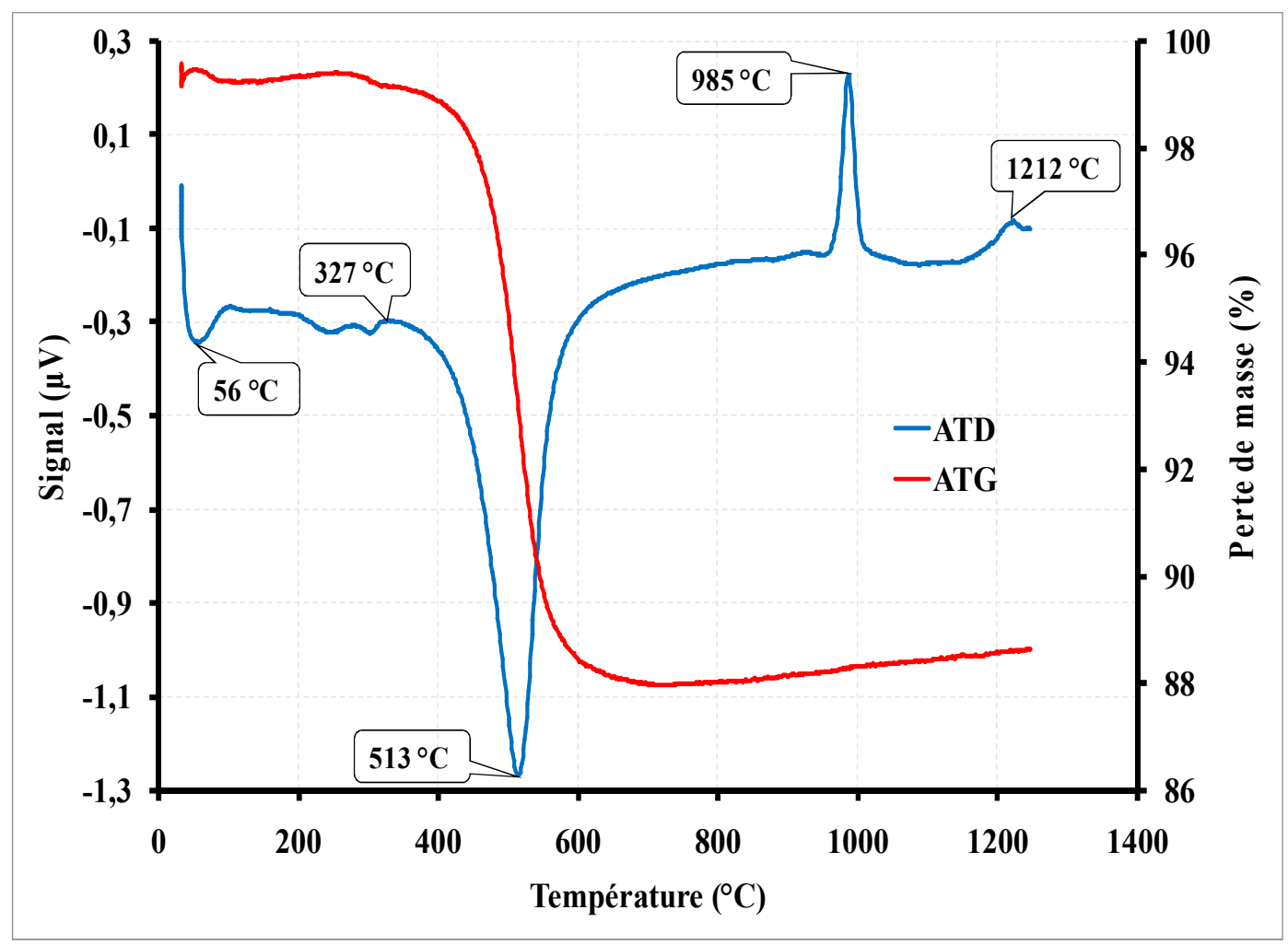

Figure 9 : Thermogrammes ATD/TG de ETI.

\section{Conclusion}

L'utilisation d'un matériau exige la maîtrise de ses propriétés physiques, chimiques, minéralogiques et géotechniques. C'est pour cela que les échantillons BAK et ETI ont été soumis à une série de caractérisation. La caractérisation géotechnique a révélé que BAK est un sol de type A2 (limono-sableux) et ETI, un sol de type A3 (argilo-limoneux). L'analyse chimique indique que ces argiles contiennent sensiblement les mêmes éléments chimiques et renferment majoritairement trois (3) oxydes à savoir la silice $\left(\mathrm{SiO}_{2}\right)$, l'alumine $\left(\mathrm{Al}_{2} \mathrm{O}_{3}\right)$ et les oxydes de fer $\left(\mathrm{Fe}_{2} \mathrm{O}_{3}\right)$. La minéralogique et les analyses thermiques ont permis d'identifier, de confirmer et d'évaluer les minéraux contenus dans les échantillons. Ainsi, il ressort de cela que la kaolinite est la minérale majoritaire dans ETI $(78,16 \%)$. Le quartz et la kaolinite représentent respectivement 49,43 et $33,11 \%$ des minéraux contenus dans BAK. La similitude de composition des différents échantillons avec les argiles utilisées dans les domaines des BTC et de la céramique montre que BAK peut être utilisés dans la formulation des briques crues et dans la confection de céramique fine (faïence, porcelaine). Il peut également servir de colorant naturel pour divers usages (coloration de tuiles, briques...) grâce à la forte teneur en oxyde de fer $(13,20 \%)$. Cependant, des études complémentaires en vue de valoriser ces grandes quantités de matières premières dans l'éco-construction et la céramique feront l'objet des études avenir en vue de trouver une solution durable et écologique pour la crise de logement qui sévit actuellement au Bénin.

\section{CONFLIT D'INTERETS}

Les auteurs déclarent qu'ils n'ont aucun conflit d'intérêt.

\section{CONTRIBUTIONS DES AUTEURS}

ABL est l'investigateur principal, il a prélevé les échantillons, réalisé les travaux, et a rédigé le manuscrit. $\mathrm{MG}, \mathrm{BS}$, ES et DKCS 
ont tous participé à la correction du manuscrit. $\mathrm{BS}$ et PB sont les initiateurs de cet article. Ils ont situé le contexte et défini l'objectif de cet article. MB a approuvé la méthode adoptée et participé à la correction du manuscrit. MG a coordonné et orienté ce travail et donner un contenu scientifique basé sur les connaissances dans ce domaine.

\section{REMERCIEMENTS}

$\mathrm{Ce}$ Travail a été financé par l'ambassade de France au Bénin (SCAC Cotonou). Les auteurs expriment leurs remerciements pour l'appui matériel et financier dont ce travail a bénéficié.

\section{RÉFÉRENCES}

Amoha BK. 2006. Coloration des blocs d'argile ciment (CIMARG). DEA Sciences de la terre, Université de Cocody, Abidjan, Côte d'Ivoire.

Ben Ayed H. 2009. Etude du béton d'argile : Application aux Briques de Terre Stabilisée. Mastère ENIT de Tunis, Tunisie.

Camille M. 2010. Contribution à la formulation et à la caractérisation d'un écomatériau de construction à base d'agroressources. Thèse de Doctorat de l'Université de Toulouse.

Ceratec. 1987. Blocs en terre stabilisée. Technologie de production.

Chenc Y, Lan GS, Tuanw H. 2000. Microstructural evolution of mullite during the sintering of kaolin powder compacts. Ceramics International, 26: 715-720.

Compaoré P. 2000. Femmes, développement et transfert de technologies. Le cas des presses à karité au Burkina Faso. Thèse de Doctorat, Université de Montréal, Département de Sociologie, Montréale.

Daot P, Hays A, Houben H, Matur S, Vitoux F. 1991. Construction en Terre par le CRATerre. Edition Parenthèses.

Diawara SO. 2009. Caractéristiques géotechniques, chimiques et mécaniques des matériaux argileux utilisés dans la construction de l'actuelle cité de Djenné : essais de renforcement. Thèse de l'Université de l'université de Bamako, Mali.
Emeruwa E, Kouadio Kpo C, Kouakou Coki H, Boffoue Oui M, Assande A, Sir O, Lenoir B. 2008. Caractérisation des argiles de la région d'Abidjan : étude comparée de quelques gîtes et leur perspective de valorisation. Rev. Ivoir. Sci. Technol., 11: 177-192.

Guerraoui F, Zamama M, Ibnoussina M. 2008. Caractérisation minéralogique et géotechnique des argiles utilisées dans la céramique de Safi (Maroc). African Journal of Science and Technology, Science and Engineering, 9(1): 1-11.

Guide du terrassement routier. 1992. Guide technique pour la réalisation des remblais et des couches de forme. . Fascicule I, principes généraux,.

Jouennec A. 1990. Traité de céramique et matériaux minéraux. Septima.

KEITA I. 2014. Géomatériaux argileux du Mali pour la construction.Propriétés mécaniques, durabilité et rôle des tanins. Thèse de Doctorat, Universite des Sciences Techniques et Technologiques de Bamako ; Universite de Limoges.

Konank L. 2006. Interaction entre des matériaux argileux et un milieu basique riche en calcium. Thèse de Doctorat Unique, Université de Limoges, France.

Laibi BA. 2008. Caractérisation physicochimique et minéralogique de quelques échantillons d'argile du bassin sédimentaire côtierdu Bénin. Mémoire de DEA, Université d'Abomey Calavi.

Ledoussalh. 1985. Les produits réfractaires. Paris: Société française de céramique.

Millogo Y. 2008. Etude géotechnique, chimique et minéralogique de matières premières argileuse et latéritique du Burkina Faso améliorées aux liants hydrauliques : application au génie civil (bâtiment et route). These de Doctorat de l'Université de Ouagadougou.

NFP18-560. 1978. Analyse granulométrique par tamisage. AFNOR.

NFP94-051. 1993. Détermination des limites d'Atterberg. AFNOR.

NFP94-057. 1992. Analyse granulométrique des sols, Méthode par sédimentation. AFNOR.

NFP94-068. 1993. Mesure de la quantité et de l'activité de la fraction argileuse. Détermination de la valeur de bleu de 
méthylène d'un sol par l'essai à la tâche. AFNOR.

NFP94-093. 1999. Détermination des références de compactage d'un matériau. AFNOR.

NFEN933-8. 1999. Essais pour déterminer les caractéristiques géométriques des granulats - Partie 8 : Évaluation des fines - Équivalent de sable. Paris: Association Française de Normalisation (AFNOR).

Njopwouo D, Orliac M. 1979. Note sur le comportement de certains minéraux à l'attaque triacide. Cah. ORSTOM - sér. pédol., XVII(4): 329-337.

Rollet AP, Bouaziz R. 1972. L'Analyse Thermique (Tome 2). Gauthier-Villars: Paris.

Soi S. 2003. Influence des ions fer sur les transformations thermiques de la kaolinite. Thèse de Doctorat Unique, Université de Limoges, France.

Sagbo E, Laibi A, Senou M, Josse R, Mensah J, Borschneck D, Noack Y. 2015. Physico-Chemical and Mineralogical Characterization of some Clays from
Coastal Sedimentary Basin of Benin used in Ceramic. Res.J.chem.sci., 5(12): 1-19.

Sorgho B. 2013. Caractérisation et valorisation de quelques argiles du Burkina Faso : application au traitement des eaux et aux géomatériaux de construction. Thèse de Doctorat, Universite de Ouagadougou.

Staljanssens M, Miuy J, Marcoen Fabry J, RasseL A. 1975. Destruction de la matière organique par calcination à basse température en vue de l'analyse minéralogique des sols. Annales de la Société Géologique de Belgique, 98: 393-403.

Wetshondo OD. 2012. Caractérisation et valorisation des matériaux argileux de la province de Kinshasa (RD Congo). Thèse de Doctorat Unique de l'Université de Liège (Belgique).

Yah OH, Fripiat JJ. 1979. Data handbook for clay minerals and other non-metallic minerals. Pergamon Press. 